\title{
Donner la parole aux personnes concernées par des maladies rares
}

\author{
Jacqueline de Sáa, Hermann Amstad ${ }^{b}$ \\ a Dre, secrétaire générale ProRaris, Berne; ${ }^{b}$ Dr, amstad-kor, Bâle
}

Le 29 février 2020, ProRaris célèbre son 10 anniversaire à Genève. ProRaris est le porte-parole des personnes atteintes de maladies rares et représente leurs intérêts auprès des autorités et du grand public. ProRaris, dans ce contexte, effectue de multiples missions qui s'inscrivent dans la durée et, pour être en mesure de répondre aux attentes, doit pouvoir élargir sa base. L'acquisition de membres sympathisants pourrait soutenir le travail de l'alliance.

Une maladie est dite rare si elle touche moins d'une personne sur 2000 dans la population. On recense à ce jour de 6000 à 8000 maladies rares, ce qui équivaut à plus d'un demi-million de personnes concernées en

\section{Plus d'un demi-million de personnes sont concernées en Suisse.}

Suisse. L'importance de ce chiffre permet la comparaison avec le diabète, une pathologie bien connue du public et qui bénéficie d'une large reconnaissance au niveau national. En revanche, on peut dire que la problématique des maladies rares n'a guère retenu l'attention en Suisse jusqu'à ces dernières années.

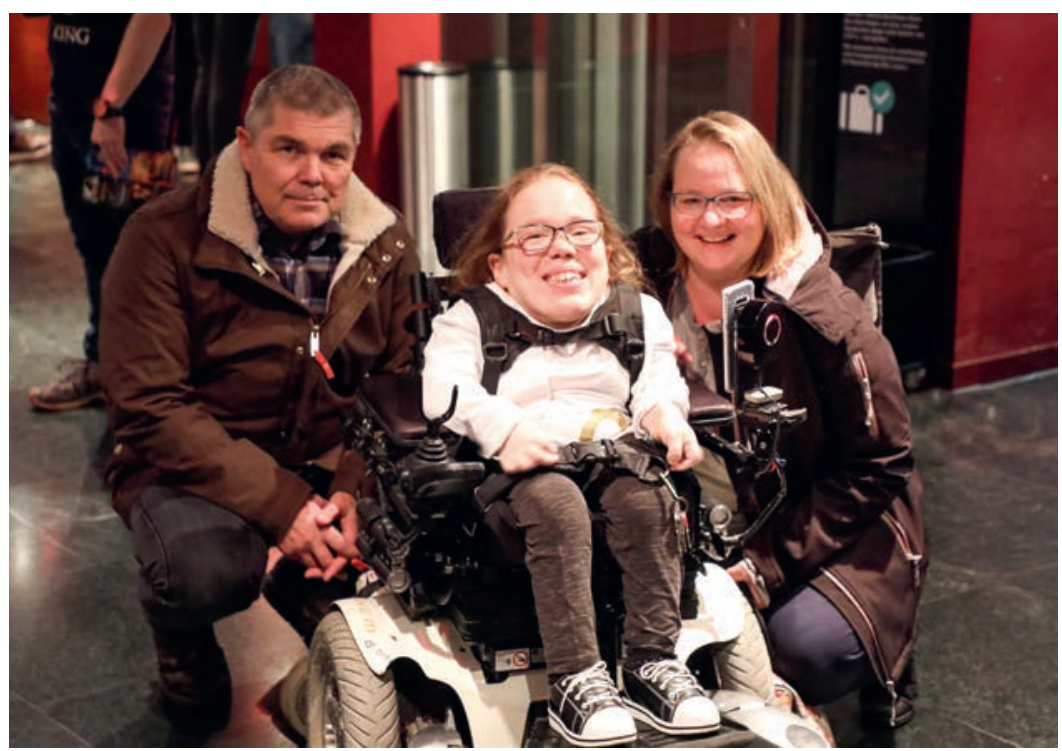

Dr Alfred Wiesbauer, vice-président de ProRaris, avec sa femme Christine et sa fille Sophie, qui souffre de mucopolysaccharidose IVa (Morbus Morquio).
En 2009, l'Union européenne a lancé une initiative pour améliorer la situation des personnes atteintes de maladies rares. Cette dynamique s'est également implantée en Suisse. En juin 2010, «ProRaris Alliance Maladies Rares - Suisse» a été fondée en qualité d'association à but non lucratif dans le but de réunir les associations de patients concernés par les maladies rares et de leur permettre de s'exprimer d'une seule voix. Aujourd'hui, l'Alliance regroupe une soixantaine d'associations de patients et de fondations dans le domaine des maladies rares.

Autre étape importante, en septembre 2018, la fondation d'UniRares, dédiée aux patients isolés qui ne bénéficient pas du soutien d'une association ou qui n'ont pas de diagnostic. ProRaris, depuis sa fondation, a toujours voulu offrir à ces nombreux patients la possibilité d'être entendus. ProRaris soutient cette jeune association sur le plan financier et administratif.

\section{Le 29 février, c'est le «Rare Disease Day»}

Eurordis, l'Organisation européenne pour les maladies rares, organise une journée annuelle des maladies rares depuis 2007, symboliquement le 29 février. En février 2011, ProRaris a organisé sa première Journée des maladies rares en Suisse. Peu de temps auparavant, le Tribunal fédéral avait rendu un arrêt qui concernait le Myozyme, un médicament orphelin destiné aux personnes souffrant de la maladie de Pompe. Une décision très controversée qui a permis aux assurances de ne plus rembourser le médicament, estimant que le rapport coût-bénéfice n'était pas respecté. Si ce jugement a gravement porté atteinte à la qualité de vie des patients concernés, il faut lui reconnaître un mérite, celui 
d'avoir porté la problématique des maladies rares sur la scène médiatique. Plus de 400 participants sont venu exprimer leur désaccord avec le Tribunal fédéral et leur soutien aux patients.

Depuis lors, ProRaris organise chaque année une journée des maladies rares. Cette journée offre aux patients, à leurs proches, aux associations de patients, aux professionnels de la santé, aux chercheurs, mais aussi aux médias et au grand public l'occasion d'échanger et de s'informer par les conférences, les tables rondes ou alors par des contacts directs.

Le 29 février 2020, la $10^{\mathrm{e}}$ Journée des maladies rares en Suisse aura lieu aux Hôpitaux Universitaires de Genève. Le programme varié présentera un bref rappel historique des débuts de ProRaris, donnera la parole aux associations de patients et définira les perspectives futures de la prise en charge des personnes concernées. L'événement est ouvert au public.

\section{Le "concept national maladies rares»: un premier succès}

Depuis sa fondation, ProRaris a développé une intense activité. En 2014, le Conseil fédéral a approuvé le concept national pour les maladies rares et, en 2015,

\section{Le thème des maladies rares a été inclus dans le catalogue des objectifs de formation de la médecine humaine.}

le plan de mise en œuvre correspondant. Le concept national a pour objectif d'assurer l'égalité d'accès au diagnostic, au traitement et aux soins pour toutes les personnes atteintes de maladies rares. En outre, les services psychosociaux destinés aux patients et à leurs proches doivent être mieux connus et plus accessibles. Un certain nombre de démarches ont déjà été mises en œuvre dans le cadre du concept national. Par exemple, le thème des maladies rares a été inclus dans le catalogue des objectifs de formation de la médecine hu-

\section{Autres liens sur les maladies rares}

\footnotetext{
- Hôpital universitaire de Zurich et Hôpital universitaire pédiatrique de Zurich: www.zentrumseltenekrankheiten.ch

- Netzwerk Rare Diseases Nordwest und Zentralschweiz: www.ukbb.ch/de/Rare-Diseases.php

- Hôpitaux universitaires de Lausanne et de Genève: www.info-maladies-rares.ch

- Malattie genetiche rare Ticino: www.malattiegeneticherare.ch

- Portail d'information: www.orpha.net

- Coordination nationale maladies rares: www.kosekschweiz.ch
}

maine et sera par la suite d'actualité dans le cadre des examens. Les travaux sur le registre suisse des maladies rares sont également bien avancés. Des Helplines spécialisées dans ce domaine ont également été mises en place en Suisse alémanique, en Suisse romande et au Tessin.

En plus de l'alliance, qualifiée de partenaire clé, de nombreux acteurs de la recherche et de la communauté médicale, ainsi que des cantons, ont participé avec beau-

\section{La Coordination nationale des maladies rares (kosek) a été fondée en juin 2017.}

coup d'engagement à la mise en œuvre. La Coordination nationale des maladies rares (kosek) a été fondée en juin 2017. ProRaris est l'un des membres fondateurs et s'est depuis impliquée avec les autres partenaires dans le développement des centres pour les maladies rares, dont l'objectif est de permettre à tous les patients concernés d'accéder aux prestations médicales. Des travaux sont également en cours sur les centres de référence spécifiques à chaque pathologie.

\section{ProRaris a besoin d'une base plus large}

Malgré ces succès, des efforts supplémentaires sont nécessaires pour garantir la pérennité et l'optimisation des structures mises en place jusqu'à présent. ProRaris reste un acteur important pour garantir que les mesures sont mises en œuvre dans le meilleur intérêt des patients. Le plus grand défi est d'ordre financier. En l'absence de base légale, il faut donc, comme indiqué dans le plan de mise en œuvre, que chaque acteur fournisse les fonds nécessaires pour les mesures qu'il entreprend lui-même. ProRaris dépend dès lors uniquement des dons pour financer ses activités (IBAN CH22 00767000 E525 2446 2, ProRaris, Vuarrens). Une autre façon de soutenir ProRaris est de devenir membre sympathisant. La participation est ouverte aux personnes juridiques ou privées qui ne remplissent pas les conditions pour devenir membre actif, mais qui souhaitent participer aux efforts pour les maladies rares. L'inscription est possible en tout temps sur le site web de ProRaris (www.proraris.ch) sous la rubrique «Membres».

Lisez également l'interview du Prof Matthias Baumgartner en pages 71-72 sur le thème des maladies rares.

Crédit photo

(c) Alfred Wiesbauer 